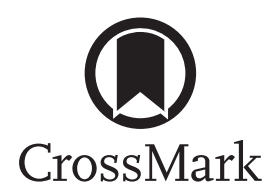

Recommending prolonged bedaquiline use for the treatment of highly resistant strains of tuberculosis

\author{
To the Editor:
}

We read with interest the article by CAMINERo et al. [1] proposing a standardised approach to treating both pre-extensively drug-resistant tuberculosis (pre-XDR-TB) and extensively drug-resistant tuberculosis (XDR-TB). This proposal is welcome considering the dearth of evidence-based recommendations on the optimal management of highly resistant tuberculosis (TB) [2]. Given the increasing recognition of the prevalence, morbidity and mortality associated with pre-XDR and XDR-TB, as well as the significant improvement in treatment outcomes with the use of novel and repurposed drugs [3], a clear policy for optimal management of these forms of TB is urgently needed.

We note that the article emphasises the use of one or both newer TB drugs, bedaquiline or delamanid, and the necessity to include them throughout the treatment or for a minimum of 13-15 months. Global discussion about the prolonged use of bedaquiline beyond 6 months for the treatment of some individuals with multidrug-resistant TB (MDR-TB) has increased [4]. Indeed, in 2017 the World Health Organization (WHO) Guidelines Development Group Meeting Report on the use of bedaquiline for the treatment of MDR-TB noted that bedaquiline has been used for longer than 6 months in some populations of limited size with no additional safety concerns being seen [5]. The accompanying frequently asked questions (FAQ) file noted that bedaquiline use beyond the 24-week recommendation in the 2013 guidelines can be considered at "...the discretion of the treating clinician..." [6]. Despite these statements, uncertainty about the prolonged use of bedaquiline has arisen amongst clinicians, technical assistance partners and national TB programmes, all of whom rely on WHO guideline updates to inform local practices and recommendations. Until additional clinical and programmatic evidence is gathered and analysed, prolonged use of bedaquiline is supported for selected individuals with MDR-TB for the following reasons: 1) The 24-week duration for bedaquiline administration was chosen to facilitate timely completion of Phase-IIb clinical trials. Additionally, extension of the corrected QT interval (QTc), the primary safety concern with bedaquiline, peaks between weeks 8 and 12 of treatment. 2) Clinical trial protocols approved by stringent regulatory authorities also allow for prolonged use of bedaquiline, even in patients without highly resistant forms of disease [7]. 3) Evidence from a cohort of people living with pre-XDR and XDR supports the efficacy and safety of prolonged bedaquiline use, suggesting a benefit in populations who have highly resistant $\mathrm{TB}$ or delayed culture conversion. Extension of bedaquiline use was associated with culture conversion in most subjects who received the drug for an extended period (median 361 days) with safety parameters comparable to those who received only 24 weeks of bedaquiline [8]. 4) There are a limited number of options available for treatment of patients with highly-resistant TB. Thus, halting a drug with both bactericidal and sterilising characteristics without having similar core drugs in the regimen to achieve a non-relapsing cure is risky. This is especially true in the case of bedaquiline, which has a prolonged terminal elimination half-life of almost 6 months. If this medication is stopped and there are not sufficient active agents left in the regimen, mycobacteria may multiply, which, in the setting of low levels of bedaquiline, could lead to the development of resistance. For patients already at high risk of treatment failure or relapse, this could leave them with few or potentially no efficacious therapeutic options. 5) The "off-label" use of medication to treat MDR-TB is common. Many types of medication, such as fluoroquinolones, linezolid and clofazimine, are recommended for use in MDR-TB without a registered indication for TB [9]. As MDR-TB is a public health emergency, such off-label use is not only clinically

@ERSpublications

Treatment of XDR-TB by prolonged use of bedaquiline is appropriate for some individuals http://ow.ly/VmpN30fzReK

Cite this article as: Furin J, Lessem E, Cox V. Recommending prolonged bedaquiline use for the treatment of highly resistant strains of tuberculosis. Eur Respir J 2017; 50: 1701552 [https://doi.org/10.1183/ 13993003.01552-2017]. 
justified but essential from a public health and ethical perspective, and as such is supported by donor funding. These same considerations must be extended to the newer TB drugs, provided the experiences and outcomes of individuals receiving them in an off-label manner are carefully documented.

This is an exciting time in the treatment of people living with MDR-TB, especially for individuals with highly resistant forms of disease. While awaiting evidence from randomised clinical trials, clinicians, policy makers and people with TB must make choices about optimal use of the newer drugs. This requires a careful analysis of risks and benefits; however, in the case of bedaquiline use beyond 24 weeks, the potential benefits will outweigh the risks for many. As always, thoughtful discussions between providers and people receiving treatment should guide choices [10]; however, it is imperative that global and national policy makers and donors support the need for prolonged use of bedaquiline in patients with MDR-TB by allowing the decision about off-label use to be made by clinicians and people receiving treatment. Such use would be facilitated by unified but flexible guidelines on MDR-TB treatment. Implementation of such comprehensive guidelines would be further strengthened by having the donor community support innovation and operational research around prolonged bedaquiline administration.

Jennifer Furin $^{1}$, Erica Lessem ${ }^{2}$ and Vivian $\operatorname{Cox}^{3}$

${ }^{1}$ Dept of Global Health and Social Medicine, Harvard Medical School, Boston, MA, USA. ${ }^{2}$ HIV/TB Project, Treatment Action Group, New York, NY, USA. ${ }^{3}$ Center for Infectious Disease Epidemiology and Research, School of Public Health and Family Medicine, University of Cape Town, Cape Town, South Africa.

Correspondence: J. Furin, 641 Huntington Ave., Boston, 02115, MA, USA. E-mail: jenniferfurin@gmail.com

Received: July 292017 | Accepted: Aug 112017

Conflict of interest: None declared.

\title{
References
}

1 Caminero JA, Piubello A, Scardigli A, et al. Proposal for a Standardised Treatment Regimen to Manage Pre- and Extensively Drug-Resistant Tuberculosis Cases. Eur Respir J 2017; 50; 1700648.

2 Lange C, Abubakar I, Alffenaar J, et al. Management of patients with multidrug-resistant/extensively drug-resistant tuberculosis in Europe: a TBNET consensus statement. Eur Respir J 2014; 44: 23-63.

3 Pontali E, Sotgiu G, D'Ambrosio L, et al. Bedaquiline and multidrug-resistant tuberculosis: a systematic and critical analysis of the evidence. Eur Respir J 2016; 47: 394-402.

4 Lewis JM, Hine P, Walker J, et al. First experience of effectiveness and safety of bedaquiline for 18 months within an optimised regimen for XDR-TB. Eur Respir J 2016; 47; 1581-1584.

5 Report of the Guideline Development Group Meeting on the use of bedaquiline in the treatment of multidrug-resistant tuberculosis. A review of available evidence (2016). Geneva, World Health Organization, 2017. http://apps.who.int/iris/ bitstream/10665/254712/1/WHO-HTM-TB-2017.01-eng.pdf?ua=1 Date last accessed: October 03, 2017.

6 Frequently asked questions about the use of bedaquiline according to the WHO guidelines and evidence from its early use in selected countries. Geneva, World Health Organization, 2017. www.who.int/tb/publications/2017/ FAQs_Bedaquiline.pdf?ua=1 Date last updated: March 13, 2017. Date last accessed: October 03, 2017.

7 STREAM: The evaluation of a standard treatment regimen of anti-tuberculosis drugs for patients with MDR-TB (NCT02409290). https://clinicaltrials.gov/ct2/show/NCT02409290?term=NCT02409290\&rank=1 Date last accessed: October 03, 2017.

8 Guglielmetti L, Jaspard M, Le Dû D, et al. Long-term outcome and safety of prolonged bedaquiline treatment for multidrug-resistant tuberculosis. Eur Respir J 2017; 49: 1601799.

9 D'Ambrosio L, Centis R, Sotgiu G, et al. New anti-tuberculosis drugs and regimens: 2015 update. ERJ Open Res 2015; 1: 00010-2015.

10 Lessem E, Cox H, Daniels C, et al. Access to new medications for the treatment of drug-resistant tuberculosis: patient, provider and community perspectives. Int J Infect Dis 2015; 32: 56-60.

Copyright (CERS 2017

\section{Bedaquiline: how better to use it}

\author{
From the authors:
}

We read with interest the correspondence by J. Furin and co-workers and we wish to thank them for their useful comments on our editorial proposing a rationale for a standardised regimen to manage difficult-to-treat cases affected by extensively drug-resistant tuberculosis (XDR-TB) or by so-called pre-extensively drug-resistant tuberculosis (pre-XDR; multidrug-resistant tuberculosis (MDR-TB) cases with additional resistance to fluoroquinolones or second-line injectable drugs) [1]. We agree with the comments by Furin and co-workers and the arguments discussing the possible use of the new drugs 
TABLE 1 Characteristics of the drugs used to treat multidrug-resistant tuberculosis and extensively drug-resistant tuberculosis

Drug

Characteristics

\begin{tabular}{lccc} 
& Bactericidal & Sterilising & Resistance prevention \\
\cline { 2 - 4 } Fluoroquinolones (moxifloxacin/gatifloxacin) & +++ & +++ & +++ \\
Bedaquiline & +++ & +++ & +++ \\
Delamanid & +++ & +++ & +++ \\
Injectables (kanamycin, amikacin, capreomycin) & +++ & + & +++ \\
Linezolid & +++ & +++ & +++ \\
High dose isoniazid & $+/+++++$ & + & +++ \\
Carbapenems limipenem/meropenem) & +++ & + & +++ \\
Clofazimine & ++++ & +++ & +++ \\
Pyrazinamide & + & +++ & + \\
Prothionamide/ethionamide & $+/++$ & $+\#$ & +++ \\
Cycloserine & + & $+\#$ & +++ \\
Para-aminosalicylic acid & + & $+\#$ & +++
\end{tabular}

\#: not fully determined/further evidence needed.

(bedaquiline and/or delamanid) for more than 6 months. Clinicians know very well how difficult it is to manage the treatment of these cases in view of their clinical complexity (with frequent adverse events), long duration and high cost [2-4]. Considering the new perspectives for treating MDR-TB and XDR-TB we fully agree that we are presently facing exciting times. We finally have a couple of new and repurposed drugs and we are slowly discovering how best to use them, as well as how effective and safe they are [4-7]. The necessary body of knowledge to support evidence-based recommendations will need time to be raised and this is reflected by the increase in new proposals and recommendations being issued [8,9]. Among the different ingredients for a potential, future standardised regimen for MDR/XDR-TB cases, the biological plausibility of the core drugs to be used (bactericidal and sterilising) is particularly important (table 1) [1]. As both bedaquiline and delamanid have excellent characteristics, both their prolonged and/ or combined use will be challenges that will need to be faced in the future. Slowly but surely new evidence is becoming available $[2,3]$ and we are confident that, given the high morbidity and mortality caused by MDR/XDR-TB, as well as the collaborative spirit animating clinicians, public health officers, policy makers, donors and members of the affected communities, better evidence will be raised to always ensure that the patient is adequately protected. Finally, the "off-label" use of drugs is sometimes necessary to manage patients for whom no other alternatives exist. Their use requires adequate capacity in terms of clinical expertise, laboratory support and infection control measures. We therefore hope that our proposal will soon be supported by the necessary evidence so as to be useful, safe and effective.

@ERSpublications

Reflections on the best possible use of bedaquiline http://ow.ly/JiFL30fBZaQ

Cite this article as: Caminero JA, Piubello A, Scardigli A, et al. Bedaquiline: how better to use it. Eur Respir J 2017; 50: 1701670 [https://doi.org/10.1183/13993003.01670-2017].

Jose A. Caminero $^{1,2}$, Alberto Piubello ${ }^{2,3}$, Anna Scardigli ${ }^{4}$ and Giovanni Battista Migliori $\odot^{5}$

${ }^{1}$ Pneumology Dept, Hospital General de Gran Canaria “Dr. Negrin”, Las Palmas de GC, Spain. ${ }^{2}$ Tuberculosis Division, International Union against Tuberculosis and Lung Disease (The Union), Paris, France. ${ }^{3}$ MDR-TB Division, Damien Foundation, Brussels, Belgium. ${ }^{4}$ The Global Fund to Fight Aids, Tuberculosis and Malaria, Geneva, Switzerland. ${ }^{5}$ Maugeri Care and Research Institute, IRCCS, Tradate, Italy.

Correspondence: Giovanni Battista Migliori, Maugeri Care and Research Institute, IRCCS, Via Roncaccio 16, 21049, Tradate, Italy. E-mail: giovannibattista.migliori@icsmaugeri.it

Received: Aug 172017 | Accepted: Aug 172017

Conflict of interest: None declared.

\section{References}

1 Caminero JA, Piubello A, Scardigli A, et al. Proposal for a standardised treatment regimen to manage pre- and extensively drug-resistant tuberculosis cases. Eur Respir J 2017; 50: 1700648. 
2 Pontali E, Sotgiu G, D'Ambrosio L, et al. Bedaquiline and multidrug-resistant tuberculosis: a systematic and critical analysis of the evidence. Eur Respir J 2016; 47: 394-402.

3 Pontali E, D'Ambrosio L, Centis R, et al. Multidrug-resistant tuberculosis and beyond: an updated analysis of the current evidence on bedaquiline. Eur Respir J 2017; 49: 1700146.

4 Borisov SE, Dheda K, Enwerem M, et al. Effectiveness and safety of bedaquiline-containing regimens in the treatment of MDR- and XDR-TB: a multicentre study. Eur Respir J 2017; 49: 1700387.

5 Tiberi S, Payen MC, Sotgiu G, et al. Effectiveness and safety of meropenem/clavulanate-containing regimens in the treatment of MDR- and XDR-TB. Eur Respir J 2016; 47: 1235-1243.

6 Tiberi S, Sotgiu G, D'Ambrosio L, et al. Comparison of effectiveness and safety of imipenem/clavulanate- versus meropenem/clavulanate-containing regimens in the treatment of MDR- and XDR-TB. Eur Respir J 2016; 47: $1758-1766$.

7 Dalcolmo M, Gayoso R, Sotgiu G, et al. Effectiveness and safety of clofazimine in multidrug-resistant tuberculosis: a nationwide report from Brazil. Eur Respir J 2017; 49: 1602445.

8 Caminero JA, Scardigli A. Classification of antituberculosis drugs: a new proposal based on the most recent evidence. Eur Respir J 2015; 46: 887-893.

9 Falzon D, Schünemann HJ, Harausz E, et al. World Health Organization treatment guidelines for drug-resistant tuberculosis, 2016 update. Eur Respir J 2017; 49: 1602308.

Copyright @ERS 2017 